\title{
Erratum to: Ultrasonography survey and thyroid cancer in the Fukushima Prefecture
}

\author{
Peter Jacob · Jan Christian Kaiser • \\ Alexander Ulanovsky
}

Published online: 14 March 2014

(c) Springer-Verlag Berlin Heidelberg 2014

\section{Erratum to: Radiat Environ Biophys \\ DOI 10.1007/s00411-013-0508-3}

During the revision of the manuscript, errors had been introduced in confidence intervals for the estimated prevalence (last column in Table 1). The correct values are shown in the table below. Correspondingly, the prevalence value for the first screening campaign should read as “...0.027\% (95\% CI $0.007 \% ; 0.069 \%) \quad \ldots$ " in the abstract, in the first paragraph of "Results", and in the last sentence of the section "Thyroid cancer prevalence" in the "Discussion".

Table 1 Expected prevalence of confirmed thyroid cancer among screened population groups targeted for the Fukushima Health Management Survey before April 2012, for the period from April 2012 to March 2013, and in the non-contaminated prefectures of Aomori, Yamanashi, and Nagasaki (Taniguchi et al. 2013)

\begin{tabular}{llll}
\hline Study group in prefecture(s) & Period of screening & $\begin{array}{l}\text { Weighted baseline rate in } \\
\text { the group }\left(\mathrm{PY}^{-1}\right)\end{array}$ & Estimated prevalence $(\%)^{\mathrm{a}}$ \\
\hline Fukushima (2011/2012) & October 2011-March 2012 & $0.267 \times 10^{-5}$ & $0.027(0.007 ; 0.069)$ \\
Fukushima (2012/2013) & April 2012-March 2013 & $0.332 \times 10^{-5}$ & $0.034(0.009 ; 0.088)$ \\
Aomori, Yamanashi and Nagasaki & November 2012-January 2013 & $0.317 \times 10^{-5}$ & $0.032(0.008 ; 0.084)$ \\
\hline
\end{tabular}

$P Y$ person years

a Arithmetic mean and $95 \%$ confidence interval

The online version of the original article can be found under doi:10.1007/s00411-013-0508-3.

P. Jacob $(\bowtie) \cdot$ J. C. Kaiser · A. Ulanovsky

Department of Radiation Sciences, Institute of Radiation

Protection, Helmholtz Zentrum München-German Research

Center for Environmental Health, Ingolstädter Landstraße 1,

85764 Neuherberg, Germany

e-mail: jacob@gsf.de 